\title{
NEONATAL LUPUS WITH COMPLETE HEART BLOCK
}

Gustavo Roberto Lourenço ${ }^{1}$,, lane Tamara Dondé ${ }^{1}$, Patrícia Milani de Moraes ${ }^{1}$, Carlos Augusto Rodrigues Padilha ${ }^{1}$, Juliana de Jesus Boscolo ${ }^{1}$, Renata Vaz de Oliveira ${ }^{1}$, Danielly Dantas Pimentel ${ }^{1}$, Maria Juliana da Silva Almeida ${ }^{1}$, Taisa Morete da Silva ${ }^{1}$

1.Faculdade de Medicina de São José do Rio Preto, São José do Rio Preto (SP), Brazil.

*Corresponding author: grlourenco@icloud.com

\section{BACKGROUND}

Neonatal lupus (NL) is an autoimmune disease associated to the presence of autoantibodies against ribonucleoproteins SSA/ Ro e SSB/La, and is characterized by isolated congenital heart block and/or cutaneous and hematological manifestations.

\section{CASE REPORT}

A 21-year-old pregnant, 25-week pregnant date was admitted to the hospital to a routine obstetric ultrasonography being noted fetal bradycardia of $48 \mathrm{bpm}$. Asymptomatic patient, with preserved obstetric physical examination, with the exception of fetal heart hate, which remained at $48 \mathrm{bpm}$. Fetal echocardiogram: total atrioventricular block. Laboratory tests: ANA 1/160 fine spelled fine, anti-Ro (SSA) $>240 \mathrm{U}$, Anti-La $22.9 \mathrm{U}$, other laboratory tests without changes. Therapy was started with dexamethasone $4 \mathrm{mg} / \mathrm{day}$ and salbutamol $6 \mathrm{mg} 6 / 6 \mathrm{~h}$, in addition to hydroxychloroquine $400 \mathrm{mg} /$ day $(4.65 \mathrm{mg} / \mathrm{kg})$, maintaining complete atrioventricular block, in addition to signs of diffuse edema. It was decided to start human immunoglobulin $70 \mathrm{~g} /$ dose every 2 weeks, maintaining the heart block. At 36 weeks gestation, the patient was hospitalized for a Cesarean delivery, but the newborn died after $48 \mathrm{~h}$ of birth.

\section{CONCLUSION}

Neonatal lupus is an autoimmune disease in which passive transfer of autoantibodies from the mother to the fetus results in fetal and neonatal disease. Several studies have established the association between anti-Ro/SSa and Anti-La/SSB antibodies and NL. Cardiac manifestations usually occur between 18-25 weeks of gestations, with a first-, second- and third-degree heart block. Advanced second- and third-degree blocks present with fetal bradycardia. The rash of NL usually comprises erythematous annular lesions or arcuate macules with slight central atrophy and raised active margins located primarily on the scalp and periorbital area. Other manifestations include asymptomatic elevated liver enzymes, mild hepatosplenomegaly, anemia and neurologic manifestations. The complete heart block is irreversible despite all therapies, but second-degree may be reversible with glucocorticoids, immune globulin and hydroxychloroquine. Beta-agonist can be use if fetal heart rate $<50 \mathrm{bpm}$. Early delivery should be avoided unless there is evidence of poor overall health, intrauterine growth restriction or hydrops.

\section{KEYWORDS}

Neonatal lupus, Complete heart block, Systemic erythematosus lupus. 